\title{
SPECTRAL RADIUS OF OPERATORS ASSOCIATED WITH DYNAMICAL SYSTEMS IN THE SPACES $C(X)$
}

\author{
KRZYSZTOF ZAJKOWSKI \\ Institute of Mathematics, University of Biatystok \\ Akademicka 2, 15-267 Biatystok, Poland \\ E-mail: kryza@math.uwb.edu.pl
}

Abstract. We consider operators acting in the space $C(X)$ ( $X$ is a compact topological space) of the form

$$
A u(x)=\left(\sum_{k=1}^{N} e^{\varphi_{k}} T_{\alpha_{k}}\right) u(x)=\sum_{k=1}^{N} e^{\varphi_{k}(x)} u\left(\alpha_{k}(x)\right), \quad u \in C(X),
$$

where $\varphi_{k} \in C(X)$ and $\alpha_{k}: X \rightarrow X$ are given continuous mappings $(1 \leq k \leq N)$. A new formula on the logarithm of the spectral radius $r(A)$ is obtained. The logarithm of $r(A)$ is defined as a nonlinear functional $\lambda$ depending on the vector of functions $\varphi=\left(\varphi_{k}\right)_{k=1}^{N}$. We prove that

$$
\ln (r(A))=\lambda(\varphi)=\max _{\nu \in \text { Mes }}\left\{\sum_{k=1}^{N} \int_{X} \varphi_{k} d \nu_{k}-\lambda^{*}(\nu)\right\},
$$

where Mes is the set of all probability vectors of measures $\nu=\left(\nu_{k}\right)_{k=1}^{N}$ on $X \times\{1, \ldots, N\}$ and $\lambda^{*}$ is some convex lower-semicontinuous functional on $\left(C^{N}(X)\right)^{\star}$. In other words $\lambda^{*}$ is the Legendre conjugate to $\lambda$.

1. Introduction. In the process of solving various mathematical and physical problems one comes across investigations of spectral properties of operators acting in spaces $F(X)$ of functions on a set $X$ and having a form

$$
A u(x)=\left(\sum_{k=1}^{N} a_{k} T_{\alpha_{k}}\right) u(x)=\sum_{k=1}^{N} a_{k}(x) u\left(\alpha_{k}(x)\right), \quad u \in F(X),
$$

where $\alpha_{k}: X \rightarrow X$ are given mappings (describing the dynamics of the system), $T_{\alpha_{k}} u(x)=u\left(\alpha_{k}(x)\right)$ are the evolution operators and $a_{k}$ are certain functions such that the operators $\left(a_{k} u\right)(x)=a_{k}(x) u(x)$ describe influences of a medium.

2000 Mathematics Subject Classification: Primary 47A10; Secondary 44A15.

Key words and phrases: spectral radius, Legendre transform.

The paper is in final form and no version of it will be published elsewhere. 
The spectral properties of the operators $A$ depend on all parameters: the spaces $F(X)$, the mappings $\alpha_{k}$ and the coefficients $a_{k}$. One of the most important characteristics of an operator is the spectral radius. We recall that the spectral radius of a bounded operator $A$ is $r(A)=\max \{|\lambda|: \lambda \in \sigma(A)\}$, where $\sigma(A)$ is the spectrum of $A$.

The operators $A$ having only one summand on the right hand side are called weighted shifts or weighted composition operators. Let $X$ be a topological compact space with a measure $\mu, F(X)=L_{p}(X, \mu), a \in C(X)$ and $A$ be a weighted shift operator

$$
A u(x)=a(x) u(\alpha(x)) .
$$

If $\alpha$ is invertible and the measure $\mu$ is $\alpha$-invariant, the following formula on the logarithm of the spectral radius holds:

$$
\ln r(A)=\max _{\nu \in \text { Mes }_{\alpha}}\left\{\int_{X} \ln |a(x)| d \nu\right\},
$$

where $M e s_{\alpha}$ is the set of all $\alpha$-invariant probability measures on $X$ (see [AL]). In the situation when the measure $\mu$ is $\alpha$-invariant and $\alpha$ is not invertible, A. Antonevich, V. Bakhtin and A. Lebedev introduced a new dynamical invariant $\tau(\nu)$ of an invariant measure $\nu$ generated by the mapping $\alpha$ and proved that

$$
\ln r(A)=\max _{\nu \in M e s_{\alpha}}\left\{\int_{X} \ln |a(x)| d \nu-\frac{\tau(\nu)}{p}\right\},
$$

also they proved that the dynamical invariant $\tau$ is a convex and lower-semicontinuous function on $M e s_{\alpha}$. This formula for the function $\ln r(A)$ is the Legendre transform of the function $\tau(\nu) / p$ (see $[\mathrm{ABL}])$.

It follows that under some assumptions we can obtain a very similar formula to (1) on the logarithm of the spectral radius of $A$, when it possesses not only one but several summands on the right hand side. To prove this formula we will need some facts of convex analysis. In the next section we recall some basic definitions and theorems connected with the Legendre transform (see $[\mathrm{ET}])$.

2. Lower-semicontinuous convex functions. We will consider functions with values in $\overline{\mathbf{R}}=[-\infty,+\infty]$ (extended real-valued functions). Let $\mathbf{V}$ be a real linear space. The function $f: \mathbf{V} \rightarrow \overline{\mathbf{R}}$ is called convex if the inequality

$$
f(t u+(1-t) v) \leq t f(u)+(1-t) f(v)
$$

holds for every $t \in[0,1]$ and all $u, v \in \mathbf{V}$ such that the right-hand side is well defined. The convex set $D(f)=\{v \in \mathbf{V}: f(v)<+\infty\}$ is called the effective domain of $f$.

Let $\mathbf{V}$ be, in addition, a topological space. The function $f: \mathbf{V} \rightarrow \overline{\mathbf{R}}$ is lowersemicontinuous on $\mathbf{V}$ if and only if the level sets $\{v \in \mathbf{V}: f(v) \leq c\}, c \in \mathbf{R}$, are closed. The lower-semicontinuous and convex functions will be the most important for us. Before we recall some theorems on such functions, we give the definitions of conjugate and biconjugate functions.

Let $\mathbf{V}$ be a linear normed space over a real field and $\mathbf{V}^{\star}$ be its conjugate space. Let $\left(v, v^{\star}\right)$ denote the value of the functional $v^{\star} \in \mathbf{V}^{\star}$ at the vector $v \in \mathbf{V}$. 
Definition 2.1. The function $f^{\star}: \mathbf{V}^{\star} \rightarrow \overline{\mathbf{R}}$ defined by

$$
f^{\star}\left(v^{\star}\right)=\sup \left\{\left(v, v^{\star}\right)-f(v): v \in \mathbf{V}\right\}, v^{\star} \in \mathbf{V}^{\star}
$$

is called the conjugate function of $f$, in the Legendre sense. The conjugate of $f^{\star}$, i.e. the function $f^{\star \star}$ on $\mathbf{V}$ defined by

$$
f^{\star \star}(v)=\sup \left\{\left(v, v^{\star}\right)-f^{\star}\left(v^{\star}\right): v^{\star} \in \mathbf{V}^{\star}\right\}, v \in \mathbf{V}
$$

is called the biconjugate of $f$.

TheOREM 2.1. Let $f: \mathbf{V} \rightarrow \overline{\mathbf{R}}$ be any function on $\mathbf{V}$. Then the functions $f^{\star}$ and $f^{\star \star}$ are always convex and lower-semicontinuous in the weak star topology of $\mathbf{V}^{\star}$ and in the weak topology of $\mathbf{V}$, respectively.

THEOREM 2.2. Let $f: \mathbf{V} \rightarrow(-\infty,+\infty]$ be any function nonidentically $+\infty$. Then $f^{\star \star}=$ $f$ if and only if $f$ is convex and lower-semicontinuous on $\mathbf{V}$.

REMARK. Let $\mathbf{V}=C(X)$. In the formula (1) the function $\ln r(A)=f(\ln |a|)$ is the Legendre transform of the function $f^{\star}(\nu)=\frac{\tau(\nu)}{p}$ when $\nu \in M e s_{\alpha}$ and $+\infty$ when $\nu \notin$ $M e s_{\alpha}$. The effective domain $D\left(f^{\star}\right)$ is contained in the set $M e s_{\alpha}$.

3. Spectral radius. Let $X$ be a topological compact space, let $\alpha_{k}: X \rightarrow X$ be given continuous mappings and $a_{k}$ be continuous positive functions on $X$. Let us consider an operator $A$ acting in the space $C(X)$ and having the form

$$
A u(x)=\left(\sum_{k=1}^{N} a_{k} T_{\alpha_{k}}\right) u(x)=\sum_{k=1}^{N} a_{k}(x) u\left(\alpha_{k}(x)\right) ; \quad u \in C(X) .
$$

If we denote the function $\ln a_{k}$ by $\varphi_{k}$, then we can rewrite the definition of $A$ as follows:

$$
A=\sum_{k=1}^{N} e^{\varphi_{k}} T_{\alpha_{k}}
$$

Notice that the functions $\varphi_{k}$ are some continuous functions on $X$. Let us define now the logarithm of the spectral radius of the operator $A$ as a functional which depends on the functions $\varphi_{k}$. Let $\lambda: C^{N}(X) \rightarrow \mathbf{R}$ be the nonlinear functional defined by

$$
\lambda(\varphi)=\ln r(A)
$$

where $A=\sum_{k=1}^{N} e^{\varphi_{k}} T_{\alpha_{k}}$ and $\varphi=\left(\varphi_{k}\right)_{k=1}^{N} \in C^{N}(X)$.

Before we formulate our main theorem, we introduce some notation.

Notation. Let $M e s$ denote the set of all probability measures on the set $X \times\{1, \ldots, N\}$, i.e. a vector of measures $\nu=\left(\nu_{k}\right)_{k=1}^{N} \in$ Mes iff $\sum_{k=1}^{N} \nu_{k}(X)=1$ and for every $k$ the measure $\nu_{k}$ is a positive measure on $X$.

MAIN TheOREm. There exists a convex lower-semicontinuous functional $\mathcal{T}$ on $M$ es such that

$$
\lambda(\varphi)=\max _{\nu=\left(\nu_{k}\right) \in M e s}\left\{\sum_{k=1}^{N} \int_{X} \varphi_{k} d \nu_{k}-\mathcal{T}(\nu)\right\} .
$$


Proof. We use the well known formula on the spectral radius

$$
r(A)=\lim _{n \rightarrow \infty}\left\|A^{n}\right\|^{\frac{1}{n}} .
$$

Since $T_{\alpha} a=(a \circ \alpha) T_{\alpha}$ and $T_{\alpha_{k}} T_{\alpha_{l}}=T_{\alpha_{k} \circ \alpha_{l}}$, the $n$-th power of $A$ is equal to

$$
A^{n} u(x)=\sum_{\xi \in M^{n}} a_{\xi}(x) u\left(\alpha^{\xi}(x)\right),
$$

where $M^{n}=\left\{\xi=\left(\xi_{i}\right)_{i=1}^{n}: \xi_{i} \in\{1, \ldots, N\}\right\}$,

$$
a_{\xi}(x)=a_{\xi_{1}}(x) a_{\xi_{2}}\left(\alpha_{\xi_{1}}(x)\right) \ldots a_{\xi_{n}}\left(\alpha_{\xi_{1}} \circ \ldots \circ \alpha_{\xi_{n-1}}(x)\right) \quad \text { and } \quad \alpha^{\xi}(x)=\alpha_{\xi_{1}} \circ \ldots \circ \alpha_{\xi_{n}}(x) \text {. }
$$

Notice that since $a_{k}$ is a positive function for every $k, a_{\xi}$ is also a positive function for every $\xi$. Therefore the norm of the $n$-th power of $A$ equals

$$
\left\|A^{n}\right\|=\sup _{X} \sum_{\xi \in M^{n}} a_{\xi}(x) .
$$

The main part of the proof is based on the following three lemmas.

Lemma 1. The functional $\lambda$ is a convex function on $C^{N}(X)$.

Proof of Lemma 1. Let $\varphi$ and $\psi$ be any vectors of functions that belong to $C^{N}(X)$. We denote $e^{\varphi_{k}}$ and $e^{\psi_{k}}$ by $a_{k}$ and $b_{k}$, respectively. Let $A$ denote the operator $\sum_{k=1}^{N} a_{k} T_{\alpha_{k}}$ and $B$ denote the operator $\sum_{k=1}^{N} b_{k} T_{\alpha_{k}}$. Let us consider the value of $\lambda$ at a vector $t \varphi+(1-t) \psi$ for some $t \in(0,1)$. Let $C_{t}$ denote the operator corresponding to the argument $t \varphi+(1-t) \psi$. We have that

$$
C_{t}=\sum_{k=1}^{N} e^{t \varphi_{k}+(1-t) \psi_{k}} T_{\alpha_{k}}=\sum_{k=1}^{N} a_{k}^{t} b_{k}^{1-t} T_{\alpha_{k}} .
$$

The $n$-th power of $C_{t}$ of a function $u \in C(X)$ at $x$ is equal to

$$
C_{t}^{n} u(x)=\left(\sum_{\xi \in M^{n}} a_{\xi}^{t} b_{\xi}^{1-t} T_{\alpha^{\xi}}\right) u(x)=\sum_{\xi \in M^{n}}\left[a_{\xi}(x)\right]^{t}\left[b_{\xi}(x)\right]^{1-t} u\left(\alpha^{\xi}(x)\right)
$$

and the norm of $C_{t}^{n}$ equals

$$
\left\|C_{t}^{n}\right\|=\sup _{X} \sum_{\xi \in M^{n}}\left[a_{\xi}(x)\right]^{t}\left[b_{\xi}(x)\right]^{1-t} .
$$

Let $t=1 / p$ and $1-t=1 / q$. Since $t \in(0,1)$, the numbers $p, q>1$ are conjugate, i.e. $1 / p+1 / q=1$. Applying the Hölder inequality for sums we get

$$
\sum_{\xi \in M^{n}}\left[a_{\xi}(x)\right]^{t}\left[b_{\xi}(x)\right]^{1-t} \leq\left[\sum_{\xi \in M^{n}} a_{\xi}(x)\right]^{t}\left[\sum_{\xi \in M^{n}} b_{\xi}(x)\right]^{1-t} .
$$

By the above

$$
\left\|C_{t}^{n}\right\| \leq\left\|A^{n}\right\|^{t}\left\|B^{n}\right\|^{1-t}
$$

and, in consequence,

$$
r\left(C_{t}\right) \leq[r(A)]^{t}[r(B)]^{1-t}
$$

Therefore we obtain

$$
\lambda(t \varphi+(1-t) \psi)=\ln r\left(C_{t}\right) \leq t \ln r(A)+(1-t) \ln r(B)=t \lambda(\varphi)+(1-t) \lambda(\psi) .
$$

This means that the functional $\lambda$ is convex on $C^{N}(X)$ and Lemma 1 follows. 
The continuity of $\lambda$ is established by our next lemma.

Lemma 2. The functional $\lambda$ is a continuous function on $C^{N}(X)$.

Proof of Lemma 2. Let $\varphi^{m}=\left(\varphi_{k}^{m}\right)_{k=1}^{N}, \varphi^{0}=\left(\varphi_{k}^{0}\right)_{k=1}^{N}$ belong to $C^{N}(X)$ and let $\varphi^{m}$ converge to $\varphi^{0}$ in $C^{N}(X)$, i.e. $\left\|\varphi^{0}-\varphi^{m}\right\|_{\infty}=\max _{1 \leq k \leq N}\left\|\varphi_{k}^{0}-\varphi_{k}^{m}\right\|_{\infty} \rightarrow 0$ when $m$ tends to infinity. Let $a_{k}^{m}$ denote the function $e^{\varphi_{k}^{m}}$. Notice that for every $k \in\{1, \ldots, N\}$ we have

$$
a_{k}^{m}(x)=e^{\left(\varphi_{k}^{m}-\varphi_{k}^{0}+\varphi_{k}^{0}\right)(x)}=e^{\left.\left(\varphi_{k}^{m}-\varphi_{k}^{0}\right)\right)(x)} a_{k}^{0}(x) .
$$

Since for every $x \in X$ the following inequalities hold:

$$
-\left\|\varphi^{m}-\varphi^{0}\right\|_{\infty} \leq\left(\varphi_{k}^{m}-\varphi_{k}^{0}\right)(x) \leq\left\|\varphi^{m}-\varphi^{0}\right\|_{\infty},
$$

it follows that for every $k$

$$
e^{-\left\|\varphi^{m}-\varphi^{0}\right\|_{\infty}} a_{k}^{0}(x) \leq a_{k}^{m}(x) \leq e^{\left\|\varphi^{m}-\varphi^{0}\right\|_{\infty}} a_{k}^{0}(x) .
$$

In consequence for every $\xi \in M^{n}$ we get that

$$
e^{-n\left\|\varphi^{m}-\varphi^{0}\right\|_{\infty}} \sum_{\xi \in M^{n}} a_{\xi}^{0}(x) \leq \sum_{\xi \in M^{n}} a_{\xi}^{m}(x) \leq e^{n\left\|\varphi^{m}-\varphi^{0}\right\|_{\infty}} \sum_{\xi \in M^{n}} a_{\xi}^{0}(x) .
$$

From this we obtain

$$
e^{-\left\|\varphi^{m}-\varphi^{0}\right\|_{\infty}}\left\|A_{0}^{n}\right\|^{\frac{1}{n}} \leq\left\|A_{m}^{n}\right\|^{\frac{1}{n}} \leq e^{\left\|\varphi^{m}-\varphi^{0}\right\|_{\infty}}\left\|A_{0}^{n}\right\|^{\frac{1}{n}}
$$

and letting $n$ tend to infinity

$$
e^{-\left\|\varphi^{m}-\varphi^{0}\right\|_{\infty}} r\left(A_{0}\right) \leq r\left(A_{m}\right) \leq e^{\left\|\varphi^{m}-\varphi^{0}\right\|_{\infty}} r\left(A_{0}\right),
$$

where the operators $A_{0}$ and $A_{m}$ correspond to $\varphi^{0}$ and $\varphi^{m}$, respectively. Now letting $m$ tend to infinity we obtain the continuity of the spectral radius of $A$ on the space $C^{N}(X)$. Consequently, we obtain the continuity of the logarithm of spectral radius, i.e. the continuity of the functional $\lambda$ on $C^{N}(X)$, and Lemma 2 follows.

The functional $\lambda$ is a convex and continuous function on $C^{N}(X)$. Theorem 2.2 gives $\lambda=\lambda^{\star \star}$. By the above we can write that

$$
\lambda(\varphi)=\sup \left\{\sum_{k=1}^{N} \int_{X} \varphi_{k} d \nu_{k}-\lambda^{\star}(\nu): \nu=\left(\nu_{k}\right)_{k=1}^{N} \in C^{N}(X)^{\star}\right\},
$$

where

$$
\lambda^{\star}(\nu)=\sup \left\{\sum_{k=1}^{N} \int_{X} \varphi_{k} d \nu_{k}-\lambda(\varphi): \varphi=\left(\varphi_{k}\right)_{k=1}^{N} \in C^{N}(X)\right\} .
$$

The effective domain of the functional $\lambda$, i.e. the set of $\varphi$ such that $\lambda(\varphi)<+\infty$, is the whole space $C^{N}(X)$. It is interesting that the effective domain of the functional $\lambda^{\star}$ is a subset of the set of all probability measures on $X \times\{1, \ldots, N\}$, i.e. $D\left(\lambda^{\star}\right) \subset M e s$.

LEMma 3. The effective domain of the functional $\lambda^{\star}$ is contained in the set Mes.

Proof of Lemma 3. For any $\varphi=\left(\varphi_{k}\right)_{k=1}^{N} \in C^{N}(X)$ and $c \in \mathbf{R}$ let $\varphi+c$ denote the vector of functions $\left(\varphi_{k}+c\right)_{k=1}^{N}$. If to the vector of functions $\varphi$ corresponds the operator $A$, then to the vector of functions $\varphi+c$ corresponds the operator $e^{c} A$. By the above, we obtain that

$$
\lambda(\varphi+c)=\ln r\left(e^{c} A\right)=c+\ln r(A)=\lambda(\varphi)+c .
$$


For every $\varphi$ and $c$ we have that

$$
\lambda^{\star}(\nu) \geq \sum_{k=1}^{N} \int_{X}\left(\varphi_{k}+c\right) d \nu_{k}-\lambda(\varphi+c)=\sum_{k=1}^{N} \int_{X} \varphi_{k} d \nu_{k}-\lambda(\varphi)+c\left(\sum_{k=1}^{N} \nu_{k}(X)-1\right) .
$$

If $\sum_{k=1}^{N} \nu_{k}(X)-1 \neq 0$ then letting $c$ tend to plus or minus infinity we can obtain that the right hand side of the above inequality tends to plus infinity. This means that the value of the function $\lambda^{\star}$ at such a vector of measures $\nu=\left(\nu_{k}\right)_{k=1}^{N}$ is equal to plus infinity.

Let us consider now a vector of measures $\nu=\left(\nu_{k}\right)_{k=1}^{N}$ in which for some $k=l$ the measure $\mu_{l}$ is not positive. For this measure there exists a function $\psi \geq 0$ such that $\int_{X} \psi d \nu_{l}<0$. Take now a vector of functions $\varphi^{0}=\left(\varphi_{k}^{0}\right)_{k=1}^{N}$ in which $\varphi_{l}^{0}=\psi$ and $\varphi_{k}^{0}=0$ when $k \neq l$. For every $c$ we have that

$$
\lambda^{\star}(\nu) \geq \sum_{k=1}^{N} \int_{X} c \varphi_{k}^{0} d \nu_{k}-\lambda\left(c \varphi^{0}\right)=c \int_{X} \psi d \nu_{l}-\lambda\left(c \varphi^{0}\right) .
$$

Let $A_{c}^{0}$ denote the operator corresponding to some function $c \varphi^{0}$, i.e. $A_{c}^{0}=\sum_{k=1}^{N} e^{c \varphi_{k}^{0}} T_{\alpha_{k}}$. Suppose now that $c$ is a negative number. For $k=l, e^{c \varphi_{l}^{0}}=e^{c \psi} \leq 1$, and for $k \neq l$, $e^{c \varphi_{k}^{0}}=1$. It follows that $\left\|A_{c}^{0}\right\| \leq N$ and, in consequence, $r\left(A_{c}^{0}\right) \leq N$. By the above, we obtain

$$
\lambda\left(c \varphi^{0}\right)=\ln r\left(A_{c}^{0}\right) \leq \ln N
$$

for every negative $c$.

Because $c \int_{X} \psi d \nu_{l}>0$ and $\lambda\left(c \varphi^{0}\right) \leq \ln N$ for every negative $c$, the right hand side of the inequality (3) tends to plus infinity letting $c$ tend to minus infinity. It gives that the value of the function $\lambda^{\star}$ is equal to plus infinity at any vector of measures in which at least one measure is not positive. Summarizing, if $\sum_{k=1}^{N} \nu_{k}(X)-1 \neq 0$ or at least one measure $\nu_{k}$ is not positive, then $\lambda^{\star}(\nu)=+\infty$. This means that $D\left(\lambda^{\star}\right) \subset$ Mes. The proof of Lemma 3 is complete.

Notice now that, first, by Lemma 3 we may restrict our search for the supremum in the formula (2) to the set Mes, second, because the set of all probability measures is weak-star compact, we may change the supremum to the maximum. For these reasons we can rewrite the formula for the function $\lambda$ as follows:

$$
\lambda(\varphi)=\max _{\nu \in \text { Mes }}\left\{\sum_{k=1}^{N} \int_{X} \varphi_{k} d \nu_{k}-\lambda^{\star}(\nu)\right\}
$$

where $M e s$ is the set of all probability measures on $X \times\{1, \ldots, N\}$. The functional $\mathcal{T}$ in the formulation of the Main Theorem is the restriction of the conjugate functional of $\lambda$ to the set Mes. The set Mes is convex. Therefore, by Theorem 2.1, the functional $\mathcal{T}$ is also convex and lower-semicontinuous on Mes.

Final REMARK. Very similar formulae on the logarithm of $r(A)$ hold when we consider the operator $A$ acting in other spaces, but the proofs must be modified. For the case of discrete spaces, it is possible to obtain, in a combinatorial way, an explicit form for $\mathcal{T}$. The above questions will be the subject of separate papers. 


\section{References}

[A] A. Alexiewicz, Functional Analysis, PWN, Warszawa, 1969 (in Polish).

[AL] A. Antonevich and A. Lebedev, Functional Differential Equations I. $C^{*}$-theory, Longman Scientific and Technical, 1994.

[ABL] A. Antonevich, V. Bakhtin and A. Lebedev, Thermodynamics and spectral radius, Nonlinear Phenom. Complex Syst. 4 (2001), 318-321.

[ET] I. Ekeland and R. Temam, Convex Analysis and Variational Problems, North-Holland, 1976. 